\title{
A Graphene based Frequency Reconfigurable Square Patch Antenna for Telecommunication Systems
}

\author{
Mohamed Amine Ksisi \\ Department of Physics, \\ University of Tunis El Manar, \\ Tunis, Tunisia \\ ksiksimedamin@gmail.com \\ Hosni Ajlani \\ Department of Physics, \\ University of Tunis El Manar, \\ Tunis, Tunisia \\ hosni.ajlani@gmail.com
}

\author{
Mohamed Karim Azizi \\ Department of Physics, \\ University of Tunis El Manar, \\ Tunis, Tunisia \\ medkarim.azizi@gmail.com \\ Ali Gharsallah \\ Department of Physics, \\ University of Tunis El Manar, \\ Tunis, Tunisia \\ ali.gharsallah@gmail.com
}

\begin{abstract}
Current research on tunable patch antennas for wireless applications has mostly focused on the dimensional variations of patch elements, such as geometry and substrate materials, using different techniques to achieve the reconfiguration. The use of different mixing materials to ensure reconfiguration and improvement of antenna performance in microwave frequencies has not yet been studied thoroughly. In this article, we consider graphene as a patch material, due to its unique chemical, mechanical, electronic, thermal and optical features, which assist in providing a highly flexible and adaptive antenna. The proposed antenna is a square plate excited by a coaxial probe, operating at a $2.45 \mathrm{GHz}$ spectrum. Adding graphene to the antenna structure and tuning its chemical potential, a frequency reconfiguration from $2.36 \mathrm{GHz}$ to $1.26 \mathrm{GHz}$ is obtained. This antenna can be deployed in many communication systems. Results demonstrate the importance of this material in the development of nanoelectronics in the future.
\end{abstract}

\section{Keywords-patch antenna; graphene; reconfiguration}

\section{INTRODUCTION}

Reconfigurable antennas have attracted the attention of many researchers due to their ability to operate at different frequencies, standards and applications, replacing the use of multiple antennas [1]. Such a multifunctional antenna that is weight-, size- and cost-saving has innovative applications [2]. The reconfiguration of an antenna is usually accomplished by readjusting its current or reconfiguring its radiating edges [3-5]. The antenna's functionality is changed by redistributing some of its properties using a variety of techniques. The techniques that have been used since the rise of reconfigurable antennas are grouped into four categories, namely electrical, mechanical, optical, and smart material [6]. The techniques based on PIN diodes [7-8], varactors [9-10] and micro electromechanical systems (RF-MEMS) [11-12] represent the electrical reconfiguration techniques, which are based on the connection and disconnection of switches that redistribute the antenna current. On the contrary, the mechanical reconfiguration technique studied in [13] is based on the antenna's structure alteration. The optical reconfiguration technique presented in [14], introduces photoconductive switch elements. Moreover, there are reconfiguration techniques based on smart materials such as ferrites, graphene [15] and liquid crystals [16-17]. Frequency reconfigurable antennas can alter their resonant frequency and several proposals have been described in [1820]. The radiation pattern reconfigurable antennas presented in [9] can change their radiation pattern while keeping the same resonant frequency. Moreover, polarization reconfigurable antennas are able to change their polarization while keeping the resonant frequency unchanged [21].

Due to the design complexity of reconfigurable antennas, many researchers are studying new techniques to enhance their performance, such as gain, stability, impedance and limit their cost, losses, weight and length. One of these rising techniques is the utilization of graphene. Thanks to its chemical, mechanical, electronic, thermal, and optical properties [22], graphene is the ideal candidate to elaborate frequency reconfigurable antennas. This $2-\mathrm{D}$ material is made of carbon atoms, arranged in honeycombs. Its thickness is of the same order as that of a carbon atom, i.e. $0.345 \mathrm{~nm}$ and it is flexible, transparent and more resistant than steel. Moreover, graphene's massless-Dirac-fermions and ultra-high electron mobility [2324] allows the development of high-speed devices [25], optical devices [26], field effect transistors [27], and frequency multipliers [28]. Its optical conductivity can be modulated by applying an external grid voltage between the graphene layer and a ground plane. Varying the applied voltage allows modifying the behavior of the graphene layer, which can pass from a dielectric behavior to a metallic behavior or vice versa [29]. In this paper, we present a graphene based frequency reconfigurable square patch antenna for multiple communication systems. A comparison between the performance of a standard antenna using a varicap diode and 
the proposed graphene-based one shows that the latter can save space and cost and also switch between different communication frequencies through the adjustment of the applied gate bias.

\section{GRAPHENE BEHAVIOR}

Graphene is a flat mono-atomic layer of carbon atoms distributed along a two-dimensional honeycomb lattice which can be modeled by a surface conductivity $\sigma[30]$. The control of electromagnetic wave propagation has attracted research interest in various applications in microwave [31] and $\mathrm{THz}$ domains [32, 33], due to its remarkable performance. Compared with metallic materials, graphene could be more efficient for use in antennas [34]. The surface conductivity of graphene is expressed by the Kubo formula as a function of frequency $f$, temperature $T$, and chemical potential $\mu_{C}$ as [35]:

$$
\begin{gathered}
\sigma(\omega)=\frac{j q_{e}^{2}(\omega-j 2 r)}{\pi h^{-2}}\left[\frac{1}{\omega-j 2 r} \int_{0}^{\infty}\left(\varepsilon \frac{\partial f_{d}(\varepsilon)}{\partial \varepsilon}-\frac{\partial f_{d}(-\varepsilon)}{\partial \varepsilon}\right) \partial \varepsilon-\right. \\
\left.\int_{0}^{\infty} \frac{f_{d}(-\varepsilon)-f_{d}(\varepsilon)}{(\omega-j 2 r)^{2}-4\left(\frac{\varepsilon}{h}\right)^{2}} \partial \varepsilon\right]
\end{gathered}
$$

where $\omega$ is the radian frequency, $\varepsilon$ is the energy, $q_{e}$ is the charge of an electron, $h$ is Planck's constant and $f_{d}$ is the Fermi-Dirac distribution described as (2):

$$
f_{d}=\left(e^{\frac{\varepsilon-\mu_{C}}{K_{B} T}}+1\right)^{-1}
$$

where $\mu_{c}$ is the chemical potential of graphene and $K_{B}$ is the Boltzmann's constant.

\section{ANTENNA DESIGN}

\section{A. Antenna Structure}

The geometry of the proposed patch antenna is shown in Figure 1. It consists of a square patch antenna printed on a CuClad substrate $1.6 \mathrm{~mm}$ thick with relative permittivity of $\varepsilon_{\mathrm{r}}=2.2$. A $39.2 \times 39.2 \mathrm{~mm}^{2}$ patch is fed by an underneath located SMA connector and an $80 \times 80 \mathrm{~mm}^{2}$ ground plane. The proposed antenna is optimized to operate at $2.45 \mathrm{GHz}$.

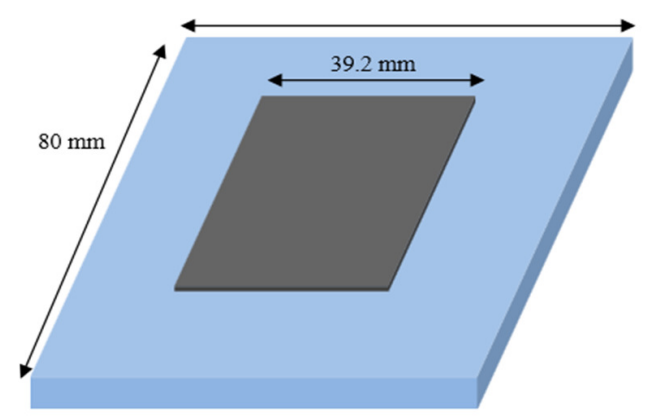

Fig. 1. Geometry of the proposed patch antenna

\section{B. Results}

The antenna structure is simulated and investigated using CST Microwave Studio. The patch antenna's behavior operating at $2.45 \mathrm{GHz}$ is studied without any additional components. The magnitude of the antenna's reflection coefficient has to be below $-10 \mathrm{~dB}$ in order to reach suitable matching impedance and is determined by Cuclad substrate. Figure 2 shows the magnitude of the antenna's reflection coefficient, with a maximum peak value of $-29.02 \mathrm{~dB}$ for the desired frequency of $2.45 \mathrm{GHz}$.

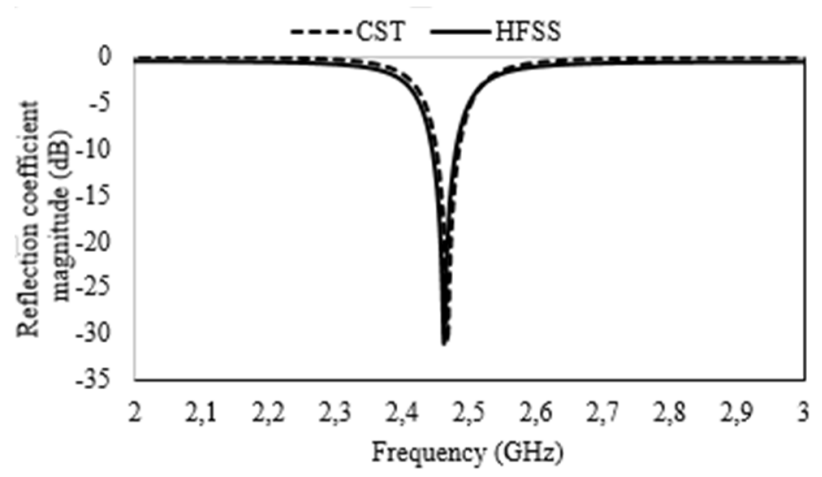

Fig. 2. Magnitude of the reflection coefficient of the initial antenna

We notice that the impedance bandwidth attained for the proposed antenna goes from 2.434 to $2.469 \mathrm{GHz}$ for $-10 \mathrm{~dB}$. For this study's simple configuration, the $-10 \mathrm{~dB}$ bandwidth is achieved with a frequency of $350 \mathrm{MHz}$. The voltage standing wave ratio (VSWR) of the antenna shown in Figure 3 describes how well the antenna gets its impedance matched, which should be less than 2 for a well efficient antenna. The proposed antenna achieves a valuable impedance matching of -29 , with $2 \mathrm{~dB}$ in the desired frequency with an ideal value of approximately 1 for the VSWR at the corresponding resonant frequency of $2.45 \mathrm{GHz}$.

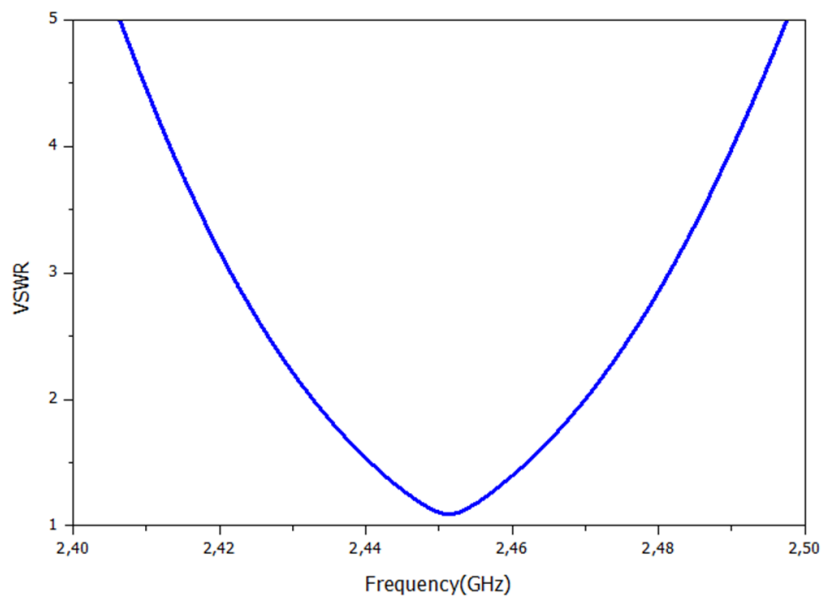

Fig. 3. VSWR

IV. THE RECONFIGURABLE ANTENNA

Using graphene as a conducting material in the antenna is anticipated to enhance both gain and bandwidth. The simulation results of the graphene antenna compared to a similar copper and varicap diode one are shown in Figure 4, indicating much better impedance matching. 


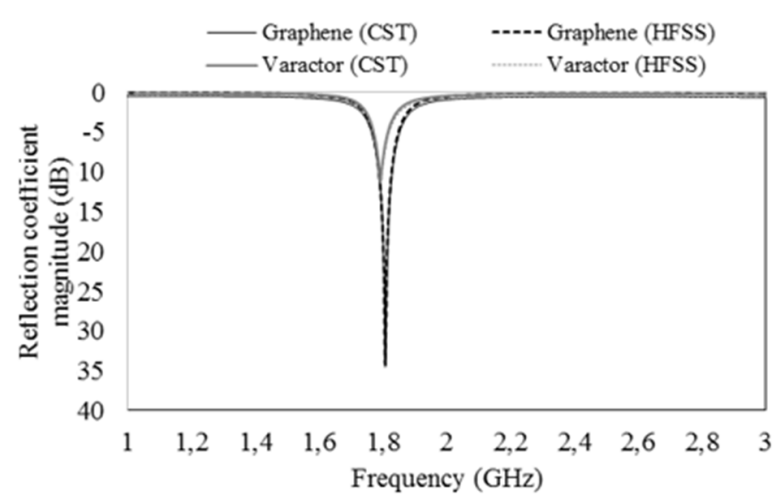

Fig. 4. Comparison between antennas with varicap diode and graphene

The antenna resonates at $1.87 \mathrm{GHz}$ for $-34 \mathrm{~dB}$ and for a chemical potential $\mu_{c}=0.3 \mathrm{eV}$. It is noticed that varying the chemical potential of the graphene antenna, a remarkable amplitude improvement is established. Moreover the graphene patch antenna achieves better impedance matching in comparison to the metallic one. Figure 5 shows the improved gain, as the graphene antenna attained $5.43 \mathrm{~dB}$ compared to the varicap diode one which attained $2 \mathrm{~dB}$.

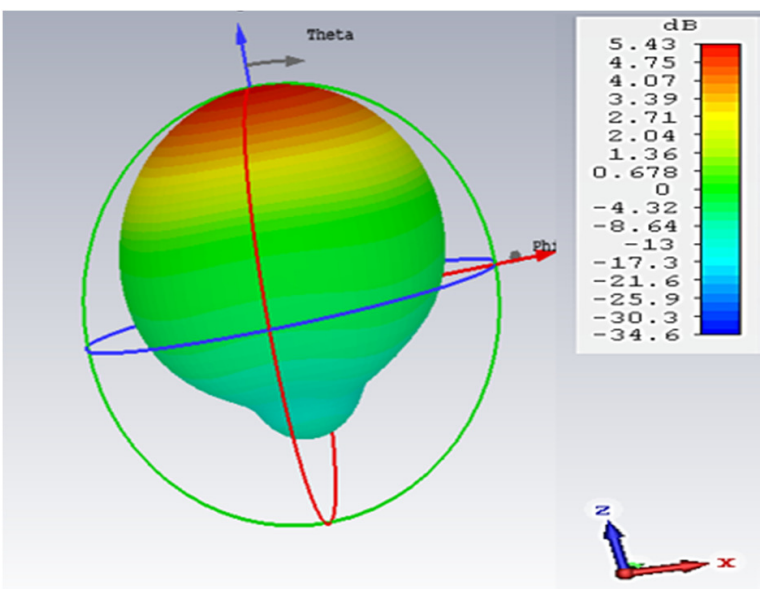

Fig. 5. Simulated 3-D gain given at $1.87 \mathrm{GHz}$ with $0.3 \mathrm{eV}$

Comparing the reconfiguration behavior of the graphene patch antenna to the metallic one using varicap diode, a simulation was carried out without any modification on the antenna's geometric dimensions [29]. Figure 6 shows better impedance matching for the graphene antenna, around $-22.14 \mathrm{~dB}$ at $1.42 \mathrm{GHz}$, which is a promising result for further practical applications. When the chemical potential is altered to $\mu_{c}=0.13 \mathrm{eV}$, another resonant peak appears with a maximum value of $-22.14 \mathrm{~dB}$ at $1.42 \mathrm{GHz}$, as shown in Figure 6 . Embedding the patch antenna with graphene leads to an improvement of $-10 \mathrm{~dB}$ in the reflection factors compared to the varicap diode one. Altering the chemical potential improves its gain, reaching about $3.76 \mathrm{~dB}$ compared to the $2 \mathrm{~dB}$ for the varicap diode one, as shown in Figure 7. The use of graphene has considerably affected the impedance properties and thus the resonant frequency of the antenna.

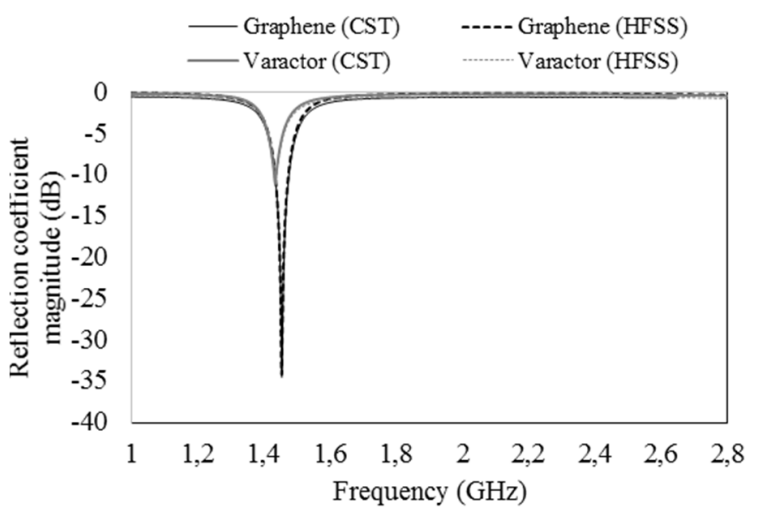

Fig. 6. The magnitude of the reflection coefficient at $1.42 \mathrm{GHz}$

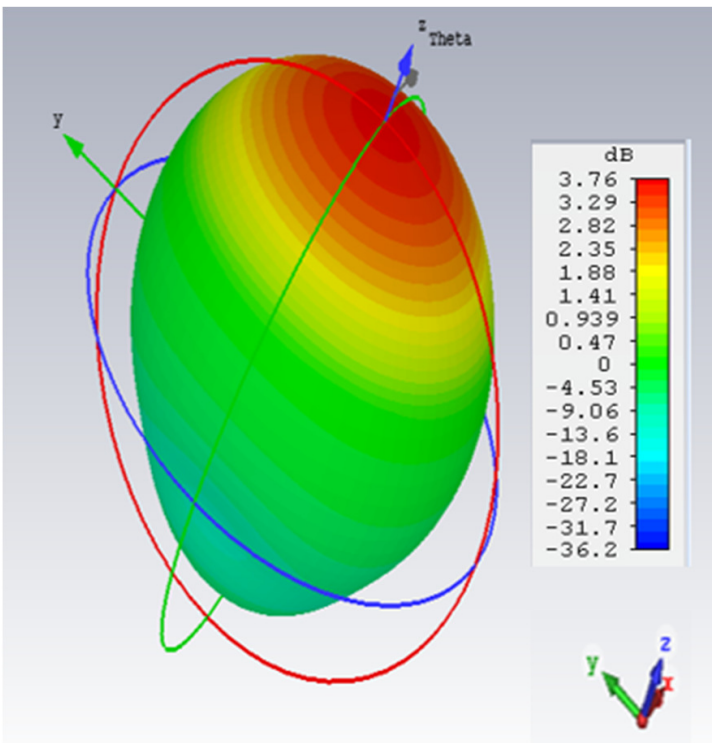

Fig. 7. Simulated 3-D gain given at $1.42 \mathrm{GHz}$

Figure 8 shows the results of using graphene instead of other techniques usually used to achieve the frequency reconfiguration of the antenna. The integration of graphene, by replacing the copper substrate used for the patch by a graphene layer, has allowed efficient control of antenna's resonant frequency. By altering the chemical potential of the graphene antenna, the resonant frequency changes, achieving frequency reconfiguration. Figure 8 exhibits the potential of using graphene to realize tunability. The antenna was perfectly adapted for different chemical potentials. Due to the use of graphene instead of varicap diode, the antenna resonates at $2.36 \mathrm{GHz}$ with a chemical potential $\mu \mathrm{c}=2 \mathrm{eV}$. By altering the graphene chemical potential from 0 to $2 \mathrm{eV}$, the antenna operates at a frequency from $1.26 \mathrm{GHz}$ to $2.36 \mathrm{GHz}$. Graphene's conductivity changes when we change its chemical potential. Therefore, a dynamic tuning of the resonant frequency of the patch antenna can be accomplished. An impedance bandwidth in the range of $300-375 \mathrm{MHz}$ is obtained for the proposed graphene patch antenna at those different resonant frequencies. 


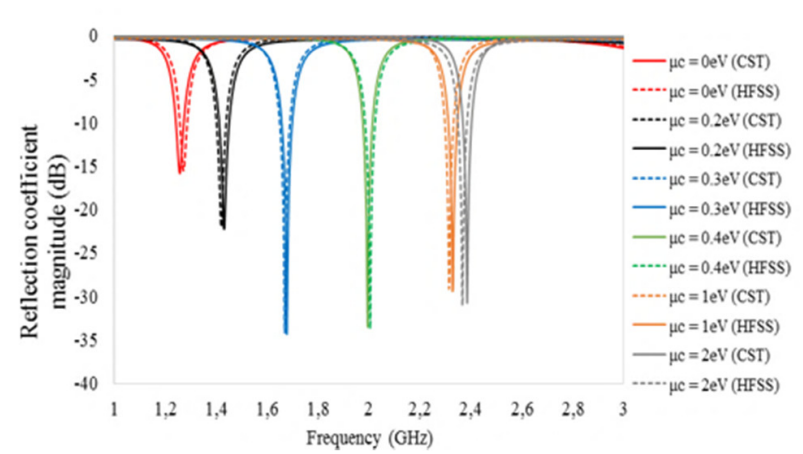

Fig. 8. Simulated S11 of the graphene antenna for different chemical potential: using HFSS and CST simulators

(a)

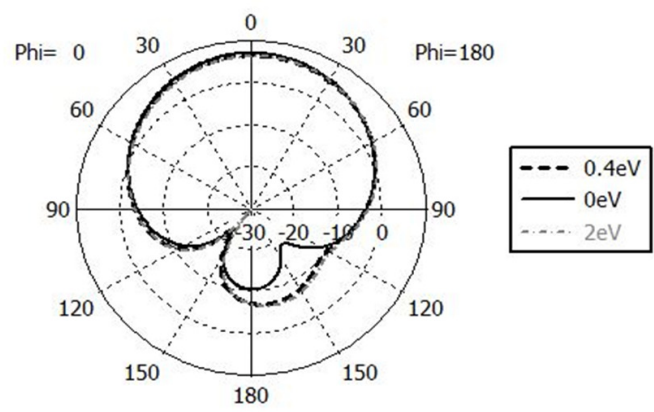

(b)

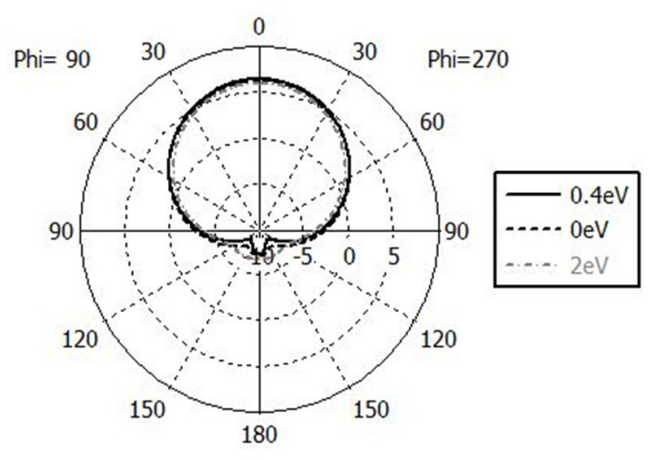

Fig. 9. Simulated (a) E- patterns (y-z plane) and (b) H- patterns (x-z plane) of the patch antenna

The E- and H-plane radiation patterns of the reconfigurable antenna presented in Figure 9 are obtained for different chemical potentials of $0 \mathrm{eV}, 0.4 \mathrm{eV}$ and $2 \mathrm{eV}$. It is clearly noted that similar broadside radiation characteristics are obtained at all E- plane patterns. Table I presents the performance comparison of this frequency reconfigurable patch antenna based on graphene with previous works [36-37]. It is clearly noted that the proposed design has widest continuous tuning range compared to these works and the highest gain value.

\section{CONCLUSION}

This paper investigated a frequency reconfigurable square patch antenna based on graphene for communication systems. The tunable resonant properties of the graphene patch antenna have been investigated in the microwave spectrum. By replacing copper in a metallic antenna with graphene, a frequency reconfiguration can be achieved with better impedance matching and remarkable gain improvement. These results show the importance of graphene as a reconfigurable rigid semi-metallic material in amplifying the antenna's gain and lowering its energy consumption. Also, graphene's particular properties proved to favor the tunability of the antenna, which is attained through an interaction between the graphene's chemical potentials. Recent research has focused on graphene as a promising material to achieve reconfiguration without any active components and to create nano-antennas through the process of miniaturization.

TABLE I. PERFORMANCE COMPARISON

\begin{tabular}{|c|c|c|}
\hline Antenna type & Fmax/Fmin (GHz) & Gain (dBi) \\
\hline $\begin{array}{c}\text { Slot loo antenna with } \\
\text { ferroelectric varactors [36] }\end{array}$ & 1.38 & 3.1 \\
\hline $\begin{array}{c}\text { Patch antenna with diode } \\
\text { varactors [37] }\end{array}$ & 1.09 & 2 \\
\hline This work & 1.87 & 3.7 \\
\hline
\end{tabular}

\section{REFERENCES}

[1] X. S. Yang, S. Q. Xiao, B. Z. Wang, "Reconfigurable Antennas", in: Compact multifunctional antennas for wireless systems, pp. 85-116, John Wiley \& Sons, 2012

[2] J. Kiriazi, H. Ghali, H. Ragaie, H. Haddara, "Reconfigurable dual-band dipole antenna on silicon using series mems switches", IEEE Antennas and Propagation Society International Symposium, Columbus, USA, June 22-27, 2003

[3] J. T. Bernhard, Reconfigurable antennas, Morgan and Claypool, 2007

[4] C. A. Balanis, Modern antenna handbook, John Wiley and Sons, 2011

[5] J. Costantine, Y. Tawk, C. G. Christodoulou, Design of reconfigurable antennas using graph models, Morgan and Claypool, 2013

[6] C. G. Christodoulou, Y. Tawk, S. A. Lane, S. R. Erwin, "Reconfigurable antennas for wireless and space applications", Procceedings of the IEEE, Vol. 100, No. 7, pp. 2250-2261, 2012

[7] P. Y. Qin, A. R. Weily, Y. J. Guo, T. S. Bird, C. H. Liang, "Frequency reconfigurable quasi-Yagi folded dipole antenna", IEEE Transactions on Antennas and Propagation, Vol. 58, No. 8, pp. 2742-2747, 2010

[8] D. Piazza, P. Mookiah, M. D’Amico, K. Dandekar, "Experimental analysis of pattern and polarization reconfigurable circular patch antennas for MIMO systems", IEEE Transactions on Vehicular Technology, Vol. 59, No. 5,pp. 2352-2362, 2010

[9] N. Behdad, K. Sarabandi, "A varactor-tuned dual-band slot antenna", IEEE Transactions on Antennas and Propagation, Vol. 54, No. 2, pp. 401-408, 2006

[10] Y. Y. Bai, S. Xiao, C. Liu, X. Shuai, B. Z. Wang, "Design of pattern reconfigurable antennas based on a two-element dipole array model", IEEE Transactions on Antennas and Propagation, Vol. 61, No. 9, pp. 4867-4871, 2013

[11] E. Erdil, K. Topalli, M. Unlu, O. A. Civi, T. Akin, "Frequency tunable patch antenna using RF MEMS technology", IEEE Transactions on Antennas and Propagation, Vol. 55, No. 4, pp. 1193-1196, 2007

[12] S. Nikolaou, N. D. Kingsley, G. E. Ponchak, J. Papapolymerou, M. M. Tentzeris, "UWB elliptical monopoles with a reconfigurable band notch using MEMS switches actuated without bias lines", IEEE Transactions on Antennas and Propagation, Vol. 57, No. 8, pp. 2242-2251, 2009

[13] S. J. Mazlouman, M. Soleimani, A. Mahanfar, C. Menon, R. G. Vaughan, "Pattern reconfigurable square ring patch antenna actuated by hemispherical dielectric elastomer", Electronics Letters, Vol. 47, No. 3, pp. 164-165, 2011

[14] L. N. Pringle, P. H. Harms, S. P. Blalock, G. N. Kiesel, E. J. Kuster, P. G. Friederich, R. J. Prado, J. M. Morris, G. S. Smith, "A reconfigurable aperture antenna based on switched links between electrically small metallic patches", IEEE Transactions on Antennas and Propagation, Vol. 52, No. 6, pp. 1434-1445, 2004 
[15] J. Perruisseau-Carrier, M. Tamagnone, J. S. Gomez-Diaz, M. EsquiusMorote, J. R. Mosig, "Resonant and leaky-wave reconfigurable antennas based on graphene plasmonics", 2013 IEEE Antennas and Propagation Society International Symposium, Orlando, USA, July 7-13, 2013

[16] L. Dixit, P. K. S. Pourush, "Radiation characteristics of switchable ferrite microstrip array antenna", IEE Proceedings - Microwaves, Antennas and Propagation, Vol. 147, No. 2, pp. 151-155, 2000

[17] W. Hu, M. Y. Ismail, R. Cahill, J. A. Encinar, V. Fusco, H. S. Gamble, D. Linton, R. Dickie, N. Grant, S. P. Rea, "Liquid-crystal-based reflectarray antenna with electronically switchable monopulse patterns", Electronics Letters, Vol. 43, No. 14, 2007

[18] K. C. Gupta, J. Li, R. Ramadoss, C. Wang, "Design of frequencyreconfigurable rectangular slot ring antennas", IEEE Antennas and Propagation Society International Symposium - Transmitting Waves of Progress to the Next Millenium, Salt Lake City, USA, July 16-21, 2000

[19] S. Xiao, B. Z. Wang, X. S. Yang, "A novel frequency-reconfigurable antenna", Microwave and Optical Technology Letters, Vol. 36, No. 4, pp. 295-297, 2003

[20] P. Y. Qin, A. R. Weily, Y. J. Guo, T. S. Bird, C. H. Liang, "Frequency reconfigurable quasi-Yagi folded dipole antenna", IEEE Transactions on Antennas and Propagation, Vol. 58, No. 8, pp. 2742-2747, 2010

[21] I. B. Trad, J. M. Floc'h, H. Rmili, M. Drissi, F. Choubani, "Rectangular bi-loop single-feed antenna with polarization agility property for GPS and iridium applications”, 2014 Loughborough Antennas Propagation Conference, Loughborough, UK, November 10-11, 2014

[22] A. K. Geim, K. S. Novoselov, "The rise of graphene", in: Nanoscience and technology: a collection of reviews from nature journals, pp. 11-19, World Scientific, 2007

[23] K. I. Bolotin, K. J. Sikes, Z. Jiang, M. Klima, G. Fudenberg, J. Hone, P. Kim, H. L. Stormer, "Ultrahigh electron mobility in suspended graphene", Solid State Communications, Vol. 146, No. 9-10, pp. 351355,2008

[24] X. Du, I. Skachko, A. Barker, E. Y. Andrei, "Approaching ballistic transport in suspended graphene", Nature Nanotechnology, Vol. 3, pp. 491-495, 2008

[25] J. S. Moon, H. C. Seo, M. Antcliffe, D. Le, C. McGuire, A. Schmitz, L. O. Nyakiti, D. K. Gaskill, P. M. Campbell, K. M. Lee, P. Asbeck, "Graphene FETs for zero-bias linear resistive FET mixers", IEEE Electron Device Letters, Vol. 34, No. 3, pp. 465-467, 2013

[26] H. Ajlani, M. K. Azizi, A. Gharsallah, M. Oueslati, "Graphene-GaAsgraphene stacked layers for the improvement of the transmission at the wavelength of 1,55 $\mu \mathrm{m}$ ", Optical Materials, Vol. 57, pp. 120-124, 2016

[27] Y. M. Lin, C. Dimitrakopoulos, K. A. Jenkins, D. B. Farmer, H. Y. Chiu, A. Grill, P. Avouris, "100-GHz transistors from wafer-scale epitaxial graphene", Science, Vol. 327, No. 5966, Article ID. 662, 2010

[28] K. S. Novoselov, A. K. Geim, S. V. Morozov, D. Jiang, Y. Zhang, S. V. Dubonos, I. V. Grigorieva, A. A. Firsov, "Electric field effect in atomically thin carbon films", Science, Vol. 306, No. 5696, pp. 666669,2004

[29] I. Rouissi, I. B. Trad, J. M. Floc'h, H. Rmili, H. Trabelsi, "Etude et conception d'une antenne patch carré reconfigurable en frequence pour les systèmes de télécommunications multistandards", XIX Journees Nationales Microondes, Bordeaux, France, June 3-5, 2015 (in French)

[30] M. Tamagnone, J. S. G. Diaz, J. Mosig, J. Perruiseau-Carrier, "Hybrid graphene-metal reconfigurable terahertz antenna", 2013 IEEE MTT-S International Microwave Symposium, Seattle, USA, June 2-7, 2013

[31] A. B. Yakovlev, G. W. Hanson, A. Mafi, "High-impedance surfaces with graphene patches as absorbing structures at microwaves", 3rd International Congress on Advanced Electromagnetic Materials in Microwaves and Optics Metamaterials, London, UK, August 30September 4, 2009

[32] C. S. R. Kaipa, A. B. Yakovlev, G. W. Hanson Y. R. Padooru, F. Medina, F. Mesa, "Enhanced transmission with a graphene-dielectric microstructure at low-terahertz frequencies", Physical Review B, Vol. 85, No. 24, Article ID 245407, 2012

[33] J. M. Jornet, I. F. Akyildiz, "Graphene-based nano-antennas for electromagnetic nanocommunications in the terahertz band", Fourth
European Conference on Antennas and Propagation, Barcelona, Spain, April 12-16, 2010

[34] K. S. Novoselov, A. K. Geim, S. V. Morosov, D. Jiang, , Y. Zhang, S. V. Dubonos, I. V. Grigorieva, A. A. Firsov, "Electric field effect in atomically thin carbon films", Science, Vol. 306, No. 5696, pp. 666669,2006

[35] G. W. Hanson, “Dyadic Green's functions for an anisotropic, non-local model of biased graphene", IEEE Transactions on Antennas and Propagation, Vol. 56, No. 3, pp. 747-757, 2008

[36] H. Y. Li, C. T. Yeh, J. J. Huang, C. W. Chang, C. T. Yu, J. S. Fu, "CPW-Fed frequency-reconfigurable slot-loop antenna with a tunable matching network based on ferroelectric varactors", IEEE Antennas and Wireless Propagation Letters, Vol. 14, pp. 614-617, 2015

[37] W. Sam, Z. Zakaria, "The investigation of the varactor diode as tuning element on reconfigurable antenna", 2016 IEEE 5th Asia-Pacific Conference on Antennas and Propagation, Kaohsiung, Taiwan, July 2629,2016 